\title{
Dynamic variability of Chinese medicine diseases and constitutions and syndromes on peritoneal cancer patients before and after cytoreductive surgery plus hyperthermic intraperitoneal chemotherapy
}

\section{Liangdeng Zhang ( $\nabla$ zldeng3@163.com )}

Capital Medical University Affiliated Beijing Shijitan Hospital https://orcid.org/0000-0003-2253-0718

\section{Ming Jiang}

Capital Medical University Affiliated Beijing Shijitan Hospital

\section{Guofeng Pan}

Capital Medical University Affiliated Beijing Shijitan Hospital

\section{Xianghua Yin}

Capital Medical University Affiliated Beijing Shijitan Hospital

\section{Research}

Keywords: Peritoneal cancer, Cytoreductive surgery plus hyperthermic intraperitoneal chemotherapy, Diseases and constitutions and syndromes change, Integrated medicine

Posted Date: May 29th, 2020

DOI: https://doi.org/10.21203/rs.3.rs-31451/v1

License: (1) This work is licensed under a Creative Commons Attribution 4.0 International License. Read Full License 


\title{
Dynamic variability of Chinese medicine diseases and constitutions and syndromes on peritoneal cancer patients before and after cytoreductive surgery plus hyperthermic intraperitoneal chemotherapy
}

Liangdeng Zhang ${ }^{1,2^{*}}$, Min Jiang ${ }^{1,2}$, Guofeng Pan ${ }^{1,2}$ and Xianghua Yin ${ }^{1,2}$

1. Department of Chinese Medicine, Beijing Shijitan Hospital, Capital Medical University, Beijing 100038

2. Beijing Cancer Institute of Integrated Medicine, Beijing Shijitan Hospital, Capital Medical University, Beijing 100038

\begin{abstract}
Background: Peritoneal cancer is advanced stage of abdominal cancer. Cytoreductive surgery (CRS) plus hyperthermic intraperitoneal chemotherapy (HIPEC) that is the main surgical regimen for peritoneal cancer patients to delay the illness. However, postoperative recovery is a long process due to extensive removal of abdominal and pelvic organs by CRS. Meanwhile, integrated Chinese and western medicine could improve life quality of cancers.
\end{abstract}

Methods: Investigating on change of disease symptoms indicators and constitution and syndrome types of peritoneal cancer patients through cross-sectional study that included three stage, before CRS plus HIPEC, within 1 week and within 3 months after CRS plus HIPEC.

Results: Total 100 patients through CRS plus HIPEC included analysis. Within 1 week after CRS plus HIPEC, both blood albumin (ALB) and haemoglobin (HGB) decreased apparently comparing to before; within 3 months after CRS plus HIPEC, ALB and HGB elevated apparently comparing 
to within 1 week; all the differences were statistically significant $(P<0.01)$. The differences of blood carbohydrate antigen (CA) 125, carcinoembryonic antigen (CEA), D-dimer (D-D), troponin I (TnI) , n-terminal precursor of brain natriuretic peptide (BNP), were statistically significant $(P<0.01)$ between before and within 1 week and 3 months after CRS plus HIPEC. The differences of both constitution and syndrome types were statistically significant $(P<0.01)$ between before and within 1 week and 3 months after CRS plus HIPEC.

Conclusions: Peritoneal cancer patients' diseases and symptoms changed apparently between before and after CRS plus HIPEC. Although, the symptomatic treatment methods by western medicine could apparently improve ALB, HGB, CA125, CEA, D-D, TnI, BNP, as well as cardiorespiratory function in a short term. Constitution groups and syndrome types of peritoneal cancer patients occurred dynamic variability between before and after CRS plus HIPEC. Generally, the primary tendency was healthy energy deficiency gradually, while pathogen excess through CRS plus HIPEC treatment. Constitutions and syndromes were mixed and complicated. Thus, to improve life quality and survival status of peritoneal cancer patients after CRS plus HIPEC, suggesting treatment integrating with Chinese medicine through the whole stage that considers diseases, symptoms, constitutions, syndromes, and the whole person.

Keywords: Peritoneal cancer, Cytoreductive surgery plus hyperthermic intraperitoneal chemotherapy, Diseases and constitutions and syndromes change, Integrated medicine

\section{Background}

Peritoneal cancer is advanced stage of abdominal cancer. Cytoreductive surgery (CRS) plus hyperthermic intraperitoneal chemotherapy (HIPEC) that is the main surgical treatment for 
peritoneal cancer to alleviate acute symptoms extensively $[1,2]$. CRS is one of the giant operation to resect abdominal and pelvic viscera involved in peritoneal cancer. HIPEC is intraoperative high dose perfusion with chemotherapy that add to treatment after CRS in the surgical process cloud be effect on logical lesions directly [3]. However, complete reduction of tumor cells is still difficult to achieve $[4]$. Peritoneal cancer belongs to abdominal mass, carcinoma, and phlegm-damp diseases in Chinese medicine. Clinical treatment with internal and external administration of Chinese medicine could relieve many cancers symptoms and syndromes, such as gastric cancer, liver cancer, mammary cancer, etc. But the effect of peritoneal cancer treatment on Chinese medicine is lack of data, as well as the whole features of Chinese medicine on diseases symptoms, constitutions, syndromes are not clear yet, especially after CRS plus HIPEC surgery. So this study was to observe clinical features of peritoneal cancer treatment on CRS plus HIPEC, including diseases symptoms, constitutions, syndromes, and the dynamic variability in Chinese medicine, which could provide clinical thinking for future integrated therapy of Chinese and western medicine.

\section{Methods}

\section{Diagnostic criteria}

Peritoneal cancer diagnostic criteria and the treatment regimen of CRS plus HIPEC referred to experts consensus [1-6]. Chinese medicine constitution diagnostic criteria referred to Chinese Society of Traditional Chinese Medicine industry standard $[7,8]$. Chinese medicine syndrome diagnostic criteria referred to the book of Diagnostics of Traditional Chinese Medicine [9], Guiding Principles for Clinical Research of New Chinese Medicine[10], Guidelines for Diagnosis and Treatment of Malignant Tumors in Traditional Chinese Medicine [11]. 


\section{Inclusion criteria}

Patients' age was over 18 years old, and informed understanding of the study, as well as signed informed consent.

\section{Exclusion criteria}

Patients diagnosed mental disease, or those who did not cooperate, or did not want to participate in the investigation were exclusion.

\section{Cases data}

The study adopted perspective design and cross-sectional method. From January 2015 to August 2019, total 100 peritoneal cancer cases from hospital inpatient of Chinese medicine ward and peritoneal cancer surgery ward were inclusion. Peritoneal cancer patients treated on CRS plus HIPEC regimen in peritoneal cancer surgery ward. All the cases treated on CRS plus HIPEC. The oldest age was 73 , youngest age 24 , average age was $(53.79 \pm 1.108)$. The longest course of peritoneal cancer was 9 years, shortest was 0.1 year, average was $(1.003 \pm 0.1262)$ years.

\section{Observation contents}

To collect general information, including sex, age, blood type, disease course, onset area, etc. Collecting dynamic follow up indexes from before CRS plus HIPEC, to within 1 week and within 3 months after CRS plus HIPEC. The follow up indexes contained tumor markers, cardiac function, blood clotting, nutrition, computed tomography (CT) examination, constitution assessment, and syndrome assessment. Tumor markers included carbohydrate antigen (CA) 125 (normal range $0 \sim 35 \mathrm{U} / \mathrm{ml}$ ), CA19-9 (normal range $0 \sim 37 \mathrm{U} / \mathrm{ml}$ ), carcinoembryonic antigen (CEA, normal range $0 \sim 5 \mathrm{ng} / \mathrm{ml}$ ), alpha fetoprotein (AFP, normal range $0 \sim 8.78 \mathrm{ng} / \mathrm{ml}$ ). Cardiac function included troponin I (TnI, normal range $0 \sim 0.04 \mathrm{ng} / \mathrm{ml}$ ), n-terminal precursor of brain natriuretic 
peptide (BNP, normal range 0 $300 \mathrm{pg} / \mathrm{ml}$ ). Blood clotting included blood D-dimer (D-D, normal range $<243 \mathrm{ng} / \mathrm{ml}$ DDU). Nutrition included blood albumin (ALB, normal range $40.0 \sim 55.0 \mathrm{~g} / \mathrm{l}$ ) and haemoglobin (HGB, normal range $115 \sim 150 \mathrm{~g} / \mathrm{l}$ ). CT examination included chest and abdomen regions. Constitution groups assessment included $q i$ deficiency, yang asthenia, yin asthenia, phlegm damp, damp heat, blood stasis, qi stagnation, special innate, and normal constitutions. Syndrome types assessment included qi-yin deficiency, yang-qi deficiency, retention of phlegm and blood stasis, stagnation of damp-heat, stagnation of $q i$ due to depression of the liver, deficiency of both the liver and kidney, deficiency of the spleen and stomach, toxin and turbid stagnation syndromes.

\section{Statistical analysis}

To use SPSS17.0 statistical package to analyze data. Measurement data indicated by (mean \pm standard deviation), and used analysis of variance or nonparametric test. Numeration data used chi-square test. The difference was statistically significant when $P<0.05$ or $P<0.01$.

\section{Results}

\section{Diseases and symptoms dynamic variation of peritoneal cancer through CRS plus HIPEC}

Total 100 peritoneal cancer patients comprised 30 male cases, while female 70 cases. All the patients were confirmed by abdominal CT examination. Rural patients were 47 cases, and urban 53 cases, using chi-square test to analyse, $\chi^{2}=0.690, P>0.05$, that was no significant difference between rural and urban area. Primary peritoneal cancer patients were 20 cases, comprising male 8 cases and female 12 cases of them. Secondary peritoneal cancer patients were 80 cases, comprising male 22 cases and female 58 cases of them. To use chi-square test to analyse, $\chi^{2}=1.190$, $P>0.05$, that was no significant difference between primary and secondary. Blood types A patients 
were 30 cases, B 33 cases, $\mathrm{AB} 5$ cases, O 32 cases, using chi-square test to analyse, $\chi^{2}=4.020$, $P>0.05$, that was no significant difference between blood types. Before CRS plus HIPEC pulmonary infection patients were 5 cases, pleural effusion 6 cases, pulmonary atelectasis 2 cases. After CRS plus HIPEC pulmonary infection patients were 26 cases, pleural effusion 28 cases, pulmonary atelectasis 31 cases, using chi-square test to analyse, $\chi^{2}=2.177, P>0.05$, that was no significant difference between pulmonary diseases and symptoms. Although pulmonary diseases and symptoms were exacerbation after CRS plus HIPEC, which could be improved obviously in a short time by western medicine symptomatic treatment. ALB used analysis of variance, $F=114.219, P=0.000$, LSD test indicated ALB decrease apparently within 1 week after CRS plus HIPEC, the difference was statistically significant comparing to before CRS plus HIPEC $(P<0.01)$. ALB increased apparently within 3 months after CRS plus HIPEC comparing to within 1 week after CRS plus HIPEC, the difference was statistically significant $(P<0.01)$. ALB was no significant difference between within 3 months after and before CRS plus HIPEC $(P>0.05)$. HGB used approximate analysis of variance, $F=193.019, p=0.000$, LSD test indicated HGB decrease apparently within 1 week after CRS plus HIPEC, the difference was statistically significant comparing to before CRS plus HIPEC $(P<0.01)$. HGB wasn't recover normal level within 3 months after CRS plus HIPEC comparing before, the difference was statistically significant $(P<0.01)$. Although HGB increased apparently within 3 months after CRS plus HIPEC comparing to within 1 week, the difference was statistically significant $(P<0.01)$. Thus, both ALB and HGB decreased severely after CRS plus HIPEC. However, western medicine symptomatic treatment included ALB infusion and blood transfusion could rapidly recover ALB and HGB. (Table 1-3)

CA125, CA19-9, CEA, AFP, D-D, TnI, BNP, all used nonparametric test to analyze. The 
difference of CA125 was statistically significant comparing between before and within 1 week and within 3 months after CRS plus HIPEC $\left(\chi^{2}=62.054, P<0.01\right)$, mean rank distribution from high to low successively that indicated the surgery program could decrease CA125 effectively. The difference of CA19-9 was no statistically significant comparing between before and within 1 week and within 3 months after CRS plus HIPEC $\left(\chi^{2}=0.754, P>0.05\right)$, mean rank distribution from high to low successively that also indicated the surgery program could decrease CA19-9 roughly. The difference of CEA was statistically significant comparing between before and within 1 week and within 3 months after CRS plus HIPEC $\left(\chi^{2}=6.499, P<0.05\right)$, mean rank distribution from high to low successively that indicated the surgery program could decrease CEA effectively, but reexamination result indicated CEA still rose again in a short period. The difference of AFP was no statistically significant comparing between before and within 1 week and within 3 months after CRS plus HIPEC $\left(\chi^{2}=4.283, P>0.05\right)$, mean rank distribution from high to low successively that indicated the surgery program could not affect AFP. The difference of D-D was statistically significant comparing between before and within 1 week and within 3 months after CRS plus HIPEC $\left(\chi^{2}=161.126, P<0.01\right)$, mean rank distribution from low to high successively that indicated the surgery program could increase D-D apparently. The difference of TnI was statistically significant comparing between before and within 1 week and within 3 months after CRS plus HIPEC $\left(\chi^{2}=13.278, P<0.01\right)$, mean rank distribution from low to high successively that indicated the surgery program could increase TnI apparently. The difference of BNP was statistically significant comparing between before and within 1 week and within 3 months after CRS plus HIPEC $\left(\chi^{2}=128.844, P<0.01\right)$, mean rank distribution from low to high successively that indicated the surgery program could increase BNP apparently. However, reexamination results indicated 
D-D, TnI, BNP, decreased again in a short period. (Table 4)

Dynamic variation of Chinese medicine constitutions and syndromes in peritoneal cancer through CRS plus HIPEC

Chinese medicine could reflect changes of the state of peritoneal cancer as the whole perspective.

Constitution groups and syndrome types used nonparametric test to analyze. The difference of constitution groups were statistically significant comparing between before and within 1 week and within 3 months after CRS plus HIPEC $\left(\chi^{2}=336.674, P<0.01\right)$, mean rank distribution from low to high successively that indicated the surgery program could increase abnormal constitution groups apparently. After operation within 1 week and 3 months the increase cases of constitution groups of $q i$ deficiency, yang asthenia, yin asthenia, phlegm damp, damp heat, blood stasis, $q i$ stagnation, special innate, comparing to before CRS plus HIPEC, while after operation the normal constitution cases decrease compared to before CRS plus HIPEC. The difference of syndrome types were statistically significant $\left(\chi^{2}=365.288, P<0.01\right)$ between before and within 1 week and 3 months after CRS plus HIPEC, including after operation within 1 week and 3 months the increase cases of syndrome types of qi-yin deficiency, yang-qi deficiency, retention of phlegm and blood stasis, stagnation of damp-heat, stagnation of $q i$ due to depression of the liver, deficiency of both the liver and kidney, deficiency of the spleen and stomach, toxin and turbid stagnation, comparing to before CRS plus HIPEC. Due to the large scale removal operation by CRS, the human body's five zang-organs and six fu-organs induced deficiency or miss. So the incoordination between the exterior and the interior had lost contact with each other. Therefore, causing Chinese medicine constitutions and syndromes to occur variation and threat the quality of life. All the patients treated on CRS plus HIPEC changing illness prognosis and causing mixed and complicated 
constitutions and syndromes. The study indicated this giant operation could lead to the positive qi deficient especially and the evil seriously through Chinese medicine constitutions and syndromes variation. (Table 5)

\section{Discussion}

Clinically, most of peritoneal cancer is secondary malignant tumor, while some of peritoneal cancer is primary in peritoneum. At present, CRS plus HIPEC therapeutic regimen is one of the mostly used operation methods for peritoneal cancer [12-14] . CRS includes a variety of surgical content in abdominal and pelvic tumor lesions removal. HIPEC is based on platinum chemotherapy regimen while implemented synchronously on operating table as CRS to jointly achieve the expectation of reducing and killing tumor cells. Postoperative recovery is a long process. The quality of life and life cycle is individual differences that is mainly related to tumor invasion and postoperative complications. Due to extensive removal of abdominal and pelvic organs by CRS, a large number of viscera are artificially caused to be absent. So postoperative complications, maintenance of nutrition, and survival status become major problems [15-17].

This study suggested that most patients with peritoneal cancer were in advanced stage at the initial diagnosis. There was no significant correlation between peritoneal cancer and the disease occurring area in rural or urban, as well as individual blood type. Thus, suggesting that the etiology of peritoneal cancer should be further studied. It's very important to move treatment forward to prevention for peritoneal cancer. Although the implementation of prevention faces many real world problems that is a key factor in the high incidence rate and mortality of cancer. Abdominal CT examination including plain three dimensional reconstruction and enhanced scanning that is the most commonly used method for the diagnosis and condition of peritoneal 
cancer. This study indicated CA125, CA19-9, CEA increased apparently before CRS plus HIPEC in peritoneal cancer patients, reflecting the high degree of tumor invasion. CA125, CA19-9, CEA decreased apparently after tumor lesions surgery removal, indicating the operation regimen was effective for improving tumor markers. ALB and HGB decreased apparently after CRS plus HIPEC in peritoneal cancer patients, due to insufficiency of circulation blood volume caused by operation regimen and the sharp consumption of nutrition. Because of the malnutrition of the patient's condition with suffer from large scale operation and chemotherapy, the whole condition changed rapidly after operation. The lung function of the patients was seriously damaged after operation that caused pulmonary infection, pulmonary atelectasis, pleural effusion. The cardiac function also was affected after operation that caused myocardial ischemia, due to long anesthesia time and long term bed rest after operation mainly. Both dysfunction of lung and heart reflected the state of complications caused by the loss of the whole structure of human body resulted by abdominal and pelvic organs resection. The balance of brain gut axis was broken by resection of large intestine and small intestine tumor lesions, affecting seriously the regulation of brain on intestine and causing intestine flora translocation. In Chinese medicine, there were interior and exterior relationship of lung and large intestine, heart and small intestine, while dysfunction of the relationship could cause comorbidity of lung and large intestine, heart and small intestine.

Peritoneal cancer patients swept and removed the abdominal and pelvic cavity multiple organs involved in tumor by CRS that caused large scale structure and function deficiency of zang-organs and $f u$-organs in Chinese medicine. The treatment regimen lasted a long time and the blood loss was too much. $Q i$ was consumed with blood to hurt primordial qi. It was easy to be attacked again by cancer poisonous pathogen. Inducing cancer metastasis and recurrence of of implant in a short 
period of time commonly. So human body constitutions and syndromes occurred dynamic change by the great trauma. However, patients' pulse condition manifested forceful pulse commonly in the postoperative stage. The clinical manifestations of forceful pulse in deficiency of both $q i$ and blood patients after CRS plus HIPEC, such as wiry pulse, large pulse, sturdy pulse, tense pulse, unsmooth pulse, etc. It was a reflection of pain in operation area and postoperative complications that indicated cancer toxin, state of evil domination symptoms. Meanwhile, patients' tongue demonstration reflected the image of spleen deficiency for long term fasting and water prohibition after operation, such as body of the tongue dark red, white and thick greasy fur on tongue, deficiency of body fluid on tongue, etc. Thus, cancer poisonous pathogen demonstrated deficiency syndrome mingling with excess syndrome frequently. Patients displayed athenia in origin and excess in superficiality. The state of evil domination reflected with pulse condition, tongue demonstration, abdominal symptoms and signs mainly. The deficiency in origin mainly contained yang $q i$ asthenia, blood deficiency, zang-organs and $f u$-organs insufficiency. The state of evil domination because of toxic and turbid connotation mainly, the specific manifestation was that endogenous phlegm, dampness, blood stasis, depression, heat, transforming into toxic turbid and block the meridian and collateral. Therefore, the basic characteristics of postoperative patients with peritoneal cancer were loss of vital energy and the accumulation of toxin and turbidity. The specific disease syndromes were judged again by the obvious domination ones of the deficiency origin or excess evil. Intestinal diseases syndromes distributed in the middle jiao and lower jiao in postoperative peritoneal cancer patients. The middle jiao and lower jiao circulated destruction while affecting heart and lung in the upper jiao inevitably. So there was a close relationship between the external and internal symptoms of lung and large intestine after the operation of 
peritoneal cancer from the perspective of triple jiao, and affected the overall body state.

This study demonstrated the dynamic evolution of peritoneal cancer patients' constitutions and syndromes before and after operative regimen. Generally, the primary tendency was healthy energy deficiency gradually, while pathogen excess through CRS plus HIPEC treatment. Constitutions and syndromes were mixed and complicated. Among nine constitution groups, $q i$ stagnation, blood stasis, phlegm damp, damp heat, were the common constitution before operation regimen. Within 1 week after operation regimen, $q i$ stagnation, $q i$ deficiency, yang asthenia, damp heat, yin asthenia, blood stasis, phlegm damp, were the common constitutions and mixed of them. Within 3 months after operation regimen, $q i$ stagnation, yin asthenia, $q i$ deficiency, damp heat, blood stasis, phlegm damp, yang asthenia, were the common constitutions and mixed of them. Before operation regimen, toxin and turbid stagnation, stagnation of $q i$ due to depression of the liver, stagnation of damp-heat, retention of phlegm and blood stasis, were the common syndromes. Within 1 week after operation regimen, stagnation of $q i$ due to depression of the liver, toxin and turbid stagnation, qi-yin deficiency, yang-qi deficiency, stagnation of damp-heat, retention of phlegm and blood stasis, deficiency of the spleen and stomach, were the common syndromes and mixed of them. Within 3 months after operation regimen, stagnation of $q i$ due to depression of the liver, toxin and turbid stagnation, stagnation of damp-heat, qi-yin deficiency, retention of phlegm and blood stasis, deficiency of the spleen and stomach, yang-qi deficiency, were the common syndromes and mixed of them.

So the treatment progress and curative effect expectation of peritoneal cancer need the integration of Chinese medicine and western medicine, giving full play to the advantage of the two medical systems. As the promotion and application of CRS plus HIPEC treatment regimen for 
peritoneal cancer and symptom therapy, leading to the dynamic evolution of diseases, symptoms, constitutions, syndromes, the treatment plan is adjusted accordingly through integrated medicine. This study demonstrated TnI, D-D, HGB, ALB, the reexamination after operation regimen could return to the normal level comparing with before operation. The reexamination of CA125, CA19-9, CEA, could decrease after operation regimen comparing with before operation. It indicated western medicine treatment was objective and effective in improving disease indicators. Although in the treatment concept of reducing and killing tumor cells is think of the body as an enemy, causing human body is deficiency of many viscera of patients. Eventually, the whole human body could not maintain its steady state. Thus, there are still many deficiencies for improving. It is urgent to innovate the treatment concept and plan, needing integrated Chinese medicine. On the treatment of Chinese medicine as a whole, starting from the dynamic changing diseases, symptoms,constitutions, syndromes, which has a certain effect on improving the overall life and living condition of patients after operation and chemotherapy. In particular, it could effectively solve the postoperative symptoms of patients with internal and external treatment of Chinese medicine $[18]$, such as oral administration of Chinese medicine, external washing of Chinese medicine, steam local therapy of Chinese medicine, acupuncture and moxibustion, etc. Therefore, the integrated treatment of Chinese medicine and western medicine is the appropriate choice for patients with peritoneal cancer after operation to harmonize human body and diseases.

\section{Conclusions}

Currently, blood indicators and CT examination, as well as others clinical examination, could be seen as diseases symptoms in modern Chinese medicine. Peritoneal cancer patients' diseases and symptoms changed apparently between before and within 1 week and 3 months after CRS plus 
HIPEC, including ALB, HGB, CA125, CEA, D-D, TnI, BNP, and cardiorespiratory function. Although, the symptomatic treatment methods by western medicine could apparently improve those indicators in a short term. However, constitution groups and syndrome types of peritoneal cancer patients occurred dynamic variability between before and within 1 week and 3 months after CRS plus HIPEC that could affect patients' postoperative state. Generally, the primary tendency was healthy energy deficiency gradually, while pathogen excess through CRS plus HIPEC treatment. Constitutions and syndromes were mixed and complicated. Thus, to improve life quality and survival status of peritoneal cancer patients after CRS plus HIPEC, suggested that treatment integrating with Chinese medicine should be through the whole stage that considers diseases, symptoms, constitutions, syndromes, and the whole person. This study provide data indicated not only removed injured organs by peritoneal cancer and reduced cancer cells by chemotherapy that seen the body diseases as enemy, but also need to be harmonious coexistence with itself.

\author{
Abbreviations \\ CRS: Cytoreductive surgery; HIPEC: Hyperthermic intraperitoneal chemotherapy; CT: Computed \\ tomography; CA: Carbohydrate antigen; CEA: Carcinoembryonic antigen; AFP: Alpha fetoprotein; \\ TnI: Troponin I; BNP: N-terminal precursor of brain natriuretic peptide; D-D: D-dimer; ALB: \\ Albumin; HGB: Haemoglobin.
}

\title{
Acknowledgements
}

The authors would like to thank peritoneal cancer surgery ward doctors and nurses for following 
up and collecting data, especially the director professor Y Li.

\section{Authors' contributions}

LD Zhang designed the study and prepared the manuscript. GF Pan, XH Yin and LD Zhang followed up and collected data. M Jiang analysed data and revised the manuscript. All authors read and approved the final manuscript.

\section{Funding}

This study was supported by Beijing Cancer Institute of Integrated Medicine Fund (No.2011), Key Specially Construction Project of the Twelfth Five Year Plan of the State Administration of Traditional Chinese Medicine (No. 2012.2)

\section{Availability of data and materials}

The data used to support the findings of this study are available from the corresponding author upon reasonable request.

\section{Ethics approval and consent to participate}

This study complied with ethical requirements of Beijing Shijitan Hospital and Capital Medical University.

\section{Consent for publication}

Not applicable. 


\section{Competing interests}

All the authors declare no conflict of interests.

\section{References}

1. Li XB, Ji ZH, Yu Y, Zhang YB, Liu G, Li B, et al. Clinical study on cytoreductive surgery plus hyperthermic intraperitoneal chemotherapy on patients with peritoneal carcinomatosis from appendiceal adenocarcinoma. J Pract Oncol. 2019;34(1):66-70.

2. Hao XS. Gide for cytoreductive surgery plus hyperthermic intraperitoneal chemotherapy in the treatment of peritoneal surface tumors expert consensus. Chi J Clin Oncol. 2015;42(4):197.

3. Li X, Ji Z, Li Y. Peritoneal carcinomatosis diagnosis and treatment in China: focusing on training and collaboration. Indian J Surg Oncol. 2019;10(Suppl 1):12-18.

4. Hotopp T. HIPEC and CRS in peritoneal metastatic gastric cancer - who really benefits? Surg Oncol. 2019;28:159-166.

5. 3. Li Y, Zhou YF, Liang H, Wang HQ, Hao JH, Zhu ZG, et al. Chinese expert consensus on cytoreductive surgery and hyperthermic intraperitoneal chemotherapy for peritoneal malignancies. World J Gastroenterol. 2016;22(30):6906-6916.

6. Li Y, Zhou YF, Liang H, Wang QH, Hao JH, Zhu ZG, et al. Expert consensus on the treatment of peritoneal surface tumor with cytoreductive surgery and intraperitoneal hyperthermic perfusion chemotherapy. Chn J Clin Oncol. 2015;42(4):198-204.

7. Chinese society of traditional Chinese Medicine. Classification and judgment of constitution of traditional Chinese Medicine (ZYYXH/T157-2009). W J Integr Trad Wes Med. 
2009;4(4):303-304.

8. Wang Q. The classification of nine basic constitution types of traditional Chinese medicine and the basis of their diagnosis. J B Univ Trad Chin Med. 2005;28(4):1-8.

9. Zhu WF. Diagnostics of traditional Chinese medicine. Beijing: China traditional Chinese Medicine Press. 2017:46-109.

10. Zheng YY. Guiding principles for clinical research of new Chinese medicine (Trial). Beijing: China Medical Science and Technology Press. 2002:207-221.

11. Lin HS. Guidelines for diagnosis and treatment of malignant tumors in traditional Chinese Medicine. Beijing: People's Health Press. 2014:36-100.

12. Ji ZH, Liang H, Ji JF, Yu Y, Li XB, Li Y. Cytoreductive surgery plus hyperthermic intraperitoneal chemotherapy on gastric cancer peritoneal carcinomatosis: a systemic analysis. Cancer Res Prev Treat. 2017;44(12):796-802.

13. Thomassen I, van Gestel YR, van Ramshorst B, Luyer MD, Bosscha K, Nienhuijis SW, et al. Peritoneal carcinomatosis of gastric origin: a population-based study on incidence, survival and risk factors. Int J Cancer. 2014;134(3):622-628.

14. Boerner T, Graichen A, Jeiter T, Zemann F, Renner P, März L, et al. CRS-HIPEC prolongs survival but is not curative for patients with peritoneal carcinomatosis of gastric cancer. Ann Surg Oncol. 2016;23(12):3972-3977.

15. Serra F, Sergi W, Spatafora F, De Ruvo N, Farinetti A, Mattioli AV, et al. Negative pressure wound therapy (NPWT) after cytoreductive surgery (CRS) and intraperitoneal chemotherapy (HIPEC) for peritoneal surface malignancies: preliminary report. G Chir. 2019;40(6):578-582.

16. Hultman B, Lind P, Glimelius B, Sundbom M, Nygren P, Haglund U, et al. Phase II study of 
patients with peritoneal carcinomatosis from gastric cancer treated with preoperative systemic chemotherapy followed by peritonectomy and intraperitoneal chemotherapy. Acta Oncol. 2013;52(4):824-30.

17. Wu HT, Yang XJ, Huang CQ, Sun JH, Ji ZH, Peng KW, et al. Cytoreductive surgery plus hyperthermic intraperitoneal chemotherapy with lobaplatin and docetaxel improves survival for patients with peritoneal carcinomatosis from abdominal and pelvic malignancies. World J Surg Oncol. 2016;14(1):246.

18. Nie B, Fu WS, Ding N, Chen J. Application of external treatment of traditional Chinese medicine in the treatment of malignant tumor. J Trad Chin Med. 2018;59(7):621-624. 
Table 1 General information

\begin{tabular}{lllll} 
& \multicolumn{2}{c}{ Sex } & Total (n) & $P$ \\
\cline { 2 - 3 } General information & Male (n) & Female (n) & \\
\hline
\end{tabular}

Area of onset

Rural

Urban

Peritoneal

Primary

Secondary

Blood type

A

B

$\mathrm{AB}$

0

Abdominal CT examination

Chest CT examination

Before operation

Pulmonary atelectasis
Pulmonary infection

Pleural effusion

2

2
16

14

8

22

11

12

0

7

30

30
31

39

12

20

80

0. 275
47

53

0.406
70

100

$19-30$

21

33

5

32

0. 239

70

100 
After operation

\begin{tabular}{lcccc} 
Pulmonary infection & 14 & 12 & 26 & \\
Pleural effusion & 11 & 17 & 28 & \\
Pulmonary atelectasis & 13 & 18 & 31 & 0.603 \\
\hline
\end{tabular}


Table 2 Follow up indicators change before and after operation regimen

Indicators Course of operation

Before operation
Within 1 week

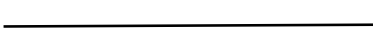

Minimum Maximum

Minimum Maximum

Mean

Minimum Maximum

Mean

Within 3 months

\begin{tabular}{|c|c|c|c|c|c|c|c|c|c|}
\hline CA125 & 5.2 & 10000.0 & $400.73 \pm 1219.71$ & 8.5 & 1192.3 & $72.43 \pm 129.15$ & 2.0 & 6763.1 & $170.14 \pm 835.61$ \\
\hline CA19-9 & 2.00 & 12000.00 & $235.43 \pm 1263.03$ & 0.92 & 1977.50 & $49.62 \pm 210.09$ & 1.70 & 6465.32 & $160.61 \pm 820.75$ \\
\hline CEA & 0.50 & 1415.68 & $28.85 \pm 144.52$ & 0.52 & 2266.65 & $35.86 \pm 244.81$ & 0.54 & 2691.83 & $37.95 \pm 271.36$ \\
\hline AFP & 1.06 & 7.97 & $2.90 \pm 1.35$ & 0.69 & 7.58 & $2.60 \pm 1.29$ & 0.61 & 7.56 & 2. $76 \pm 1.43$ \\
\hline ALB & 26.60 & 49.00 & $36.74 \pm 4.91$ & 11.10 & 39.80 & $27.73 \pm 5.47$ & 24. 60 & 46.50 & $37.23 \pm 4.59$ \\
\hline HGB & 74 & 166 & $119.98 \pm 17.61$ & 55 & 118 & $89.35 \pm 12.43$ & 73 & 147 & $113.35 \pm 16.46$ \\
\hline $\operatorname{TnI}$ & 0.01 & 0.73 & $0.02 \pm 0.07$ & 0.01 & 1. 11 & $0.04 \pm 0.14$ & 0.01 & 0.10 & $0.01 \pm 0.01$ \\
\hline BNP & 3.98 & 815.30 & $101.97 \pm 145.29$ & 8.01 & 4429. 00 & $828.29 \pm 794.13$ & 5.09 & 10584.00 & $335.32 \pm 1260.30$ \\
\hline D-D & 27 & 3669 & $557.08 \pm 572.75$ & 367 & 10292 & $3462.41 \pm 1937.47$ & 23 & 5007 & $758.25 \pm 986.47$ \\
\hline
\end{tabular}

Notes: CA125, normal range $0 \sim 35 \mathrm{U} / \mathrm{ml}$; CA19-9, normal range $0 \sim 37 \mathrm{U} / \mathrm{ml}$; CEA, normal range $0 \sim 5 \mathrm{ng} / \mathrm{ml}$; AFP, normal range $0 \sim 8.78 \mathrm{ng} / \mathrm{ml}$; TnI, normal range $0 \sim 0.04 \mathrm{ng} / \mathrm{ml} ; \mathrm{BNP}$, normal range $0 \sim 300 \mathrm{pg} / \mathrm{ml}$; D-D, normal range $<243 \mathrm{ng} / \mathrm{ml}$ DDU; HGB, normal range $115 \sim 150$ $\mathrm{g} / 1$; ALB, normal range $40.0 \sim 55.0 \mathrm{~g} / 1$ 
Table 3 ALB and HGB change before and after operation regimen

Indicators

\section{Before operation Within 1 week Within 3 months}

\begin{tabular}{|c|c|c|c|c|c|c|}
\hline $\operatorname{ALB}(g / 1)$ & $36.74 \pm 4.91$ & $27.73 \pm 5.47^{\sharp}$ & $37.23 \pm 4.59^{\text {tᄎ } \Delta}$ & $0.000^{\#}$ & $0.000^{\text {负 }}$ & $0.483^{\triangle}$ \\
\hline HGB (g/1) & $119.98 \pm 17.61$ & $89.35 \pm 12.43^{\circ}$ & $113.35 \pm 16.46^{\star \star}$ & $0.000^{\circ}$ & $0.000^{\triangle}$ & $0.003^{\star}$ \\
\hline
\end{tabular}

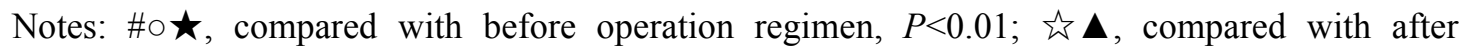
operation regimen within 1 week, $P<0.01 ; \triangle$, compared with before operation regimen, $P>0.05$ 
Table 4 CA, TnI, BNP change before and after operation regimen

Indicators

Course of operation

$\chi^{2} \quad P$

\section{Before operation Within 1 week Within 3 months}

$\begin{array}{lllccc}\text { CA125 } & 400.73 \pm 1219.71 & 72.43 \pm 129.15^{\#} & 170.14 \pm 835.61^{\text {方 }} & 62.054 & 0.000 \\ \text { CA19-9 } & 235.43 \pm 1263.03 & 49.62 \pm 210.09 & 160.61 \pm 820.75 & 0.754 & 0.686 \\ \text { CEA } & 28.85 \pm 144.52 & 35.86 \pm 244.81^{\circ \Delta} & 37.95 \pm 271.36 & 6.499 & 0.039 \\ \text { AFP } & 2.90 \pm 1.35 & 2.60 \pm 1.29 & 2.76 \pm 1.43 & 4.283 & 0.118 \\ \text { D-D } & 557.08 \pm 572.75 & 3462.41 \pm 1937.47^{\star \star} 758.25 \pm 986.47 & 161.126 & 0.000 \\ \text { TnI } & 0.02 \pm 0.07 & 0.04 \pm 0.14^{\star} & 0.01 \pm 0.01 & 13.278 & 0.001 \\ \text { BNP } & 101.97 \pm 145.29 & 828.29 \pm 794.13^{*} & 335.32 \pm 1260.30^{\bullet \bullet} 128.844 & 0.000\end{array}$

Notes: CA125, normal range $0 \sim 35 \mathrm{U} / \mathrm{ml}$; CA19-9, normal range $0 \sim 37 \mathrm{U} / \mathrm{ml}$; CEA, normal range $0 \sim 5 \mathrm{ng} / \mathrm{ml}$; AFP, normal range $0 \sim 8.78 \mathrm{ng} / \mathrm{ml}$; TnI, normal range $0 \sim 0.04 \mathrm{ng} / \mathrm{ml}$; BNP, normal range $0 \sim 300 \mathrm{pg} / \mathrm{ml}$; D-D, normal range $<243 \mathrm{ng} / \mathrm{ml} \mathrm{DDU} ; \# \star \diamond \aleph \bullet$, compared with before operation regimen, $P<0.01$; $\circ$, compared with before operation regimen, $P<0.05$; compared with after operation regimen within 1 week, $P<0.01 ; \boldsymbol{\Delta} \bullet$, compared with after operation regimen within 3 months, $P<0.01 ; \triangle$, compared with after operation regimen within 3 months, $P<0.05$ 
Table 5 Constitutions and syndromes change before and after operation regimen

Types

Before Within 1w Within 3m

Constitutions

Qi deficiency

Yang asthenia

Yin asthenia

Phlegm damp

Damp heat

Blood stasis

Qi stagnation

Special innate

Normal

Syndromes

Qi-yin deficiency

Yang-qi deficiency

Retention of phlegm and blood stasis

Toxin and turbid stagnation

Stagnation of damp-heat

Stagnation of $q i$ due to depression of the liver 15

$7 \quad 30 \quad 33$

$8 \quad 12 \quad 16$

$9 \quad 14 \quad 29$

$17 \quad 21 \quad 24$

$22 \quad 27 \quad 31$

$11 \quad 13 \quad 15$

$15 \quad 46 \quad 58$

$\begin{array}{lll}9 & 12 & 13\end{array}$

$\begin{array}{lll}2 & 1 & 1\end{array}$

0.000 
Deficiency of both the liver and kidney 